\title{
Comment on Guo et al. entitled "A prospective randomized controlled study on the treatment outcome of SpineCor brace versus rigid brace for adolescent idiopathic scoliosis with follow-up according to the SRS standardized criteria"
}

\author{
Tim Cook
}

Received: 2 April 2014/Revised: 4 May 2014/Accepted: 4 May 2014/Published online: 23 May 2014

(C) Springer-Verlag Berlin Heidelberg 2014

\section{Dear Sir}

We are writing to you to express our significant concerns about the methodology and validity of one of the articles published in your online journal on 31 December 2013 titled; "A prospective randomized controlled study on the treatment outcome of SpineCor brace versus rigid brace for adolescent idiopathic scoliosis with follow-up according to the SRS standardized criteria" [1]. It is presented as a comparison of the treatment outcomes in users of the SpineCor brace with the users of a generic rigid brace treatment.

The SpineCor brace is a clinical tool, like all other tools, that requires correct application and management to avoid poor treatment outcomes. SpineCor treatment itself is complex and requires a significant amount of training input to learn how to apply the brace correctly and how to manage the patient for the duration of the treatment.

SpineCor brace provider training involves an extensive process of different steps that not only include the initial training but also a long period of ongoing clinical support until the point at which a satisfactory level of competence is achieved.

We are astonished by the fact that none of the authors of this article, or a previous publication in 2008 showing the initial results of the same cohort of patients [2], received any brace provider training whatsoever. This must produce a strong bias to the results of their study.

Evidence of the author's lack of ability and understanding of the SpineCor brace is shown in the patient photo included in the initial publication of the same cohort of patients, where the brace had clearly been fitted

T. Cook $(\bowtie)$

The Spine Corporation Limited, Millennium House, Peak Business Park, Foxwood Road, Chesterfield S41 9RF, UK e-mail: t.cook@spinecor.com incorrectly [2]. The photo provides proof that patients in the trial were not wearing complete braces by showing the absence of the thigh bands. The thigh bands are of utmost importance for stabilising the lower 'pelvic base' section of the brace. Without the thigh bands the pelvic base will migrate upwards and loosen, which removes the effectiveness of the upper corrective part of the brace.

During the long-term treatment of a patient the replacement of brace component parts is required according to growth, wear and tear, and the loss of elasticity of the corrective part of the brace. The historical records that we have access to show that not enough brace components were provided for the braces to be well maintained for the duration of treatment.

Fundamental to any clinical trial that aims to verify the efficacy of any brace treatment is the competence of the brace provider, the correct use of the brace and the correct long-term management of the brace. The above mentioned trial has fallen short of that and therefore the validity of the results presented within it is compromised.

Conflict of interest The author represents the training/educational department of The Spine Corporation limited, who provide training to healthcare professionals in the use of the SpineCor brace.

\section{References}

1. Guo J, Lam TP, Wong MS, Ng B, Lee KM, Liu KL, Hung LH, Lau A, Sin SW, Kwok W, Yu F, Qiu Y, Cheng J (2013) A prospective randomized controlled study on the treatment outcome of SpineCor brace versus rigid brace for adolescent idiopathic scoliosis with follow-up according to the SRS standardized criteria. Eur Spine J. doi:10.1007/s00586-013-3146-1

2. Wong MS, Cheng JC, Lam TP, Ng BK, Sin SW, Lee-Shum S, Chow D, Tam S (2008) The effect of rigid versus flexible spinal orthosis on the clinical efficacy and acceptance of the patients with adolescent idiopathic scoliosis. Spine 33(12):1360-1365 